\title{
Zum Tode von Dr. Walter Bosshard
}

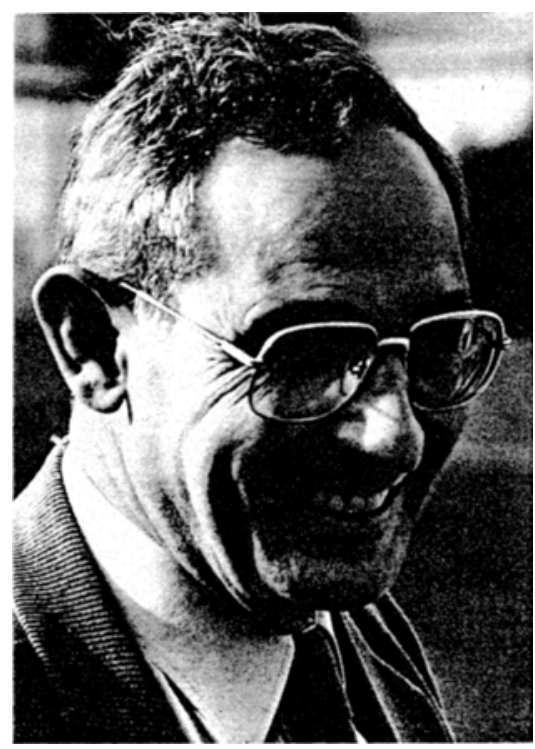

Am 1. 10. 1986 verstarb auf einer Studienreise in Polen, wenige Wochen vor seinem 60. Geburtstag, ganz unerwartet Dr. WALter Bosshard, Direktor der Eidgenössischen Anstalt für das forstliche Versuchswesen in Birmensdorf/Schweiz.

Walter Bosshard studierte in den Jahren 1947-1953 an der Forstschule der ETH Zürich. Die folgenden 3 Jahre war er Assistent von Professor Dr. H. LelBuNDGLT am damaligen Institut für Waldbau. Während der Jahre 1956-1958 arbeitete der junge Forstingenieur als Experte der FAO in Äthiopien. 1959 kehrte er nach Zürich zurück und verfaßte unter der Leitung von Professor Dr. H. Lerblivdout seine Dissertation. Noch einmal, von 1963 bis 1967, war W BOSSHARD als Experte der FAO im Sudan tätig. Anschließend übernahm er eine neue Aufgabe im Basler Chemiekonzern Geigy.

Auf den 1. Oktober 1969 wurde er als Direktor an die Eidgenössische Anstalt für das forstliche Versuchswesen gewählt; auf den Tag genau 17 Jahre hat er dieser Anstalt vorgestanden, eine Aufgabe, die ihn erfüllt hat und der er mit dem ihm eigenen Engagement nachgegangen ist.

WALTER BOSSHARD war ein Mensch mit vielseirigen Anlagen, ohne Hang und Neigung zu eingleisiger Spezialisierung und mit dem sicheren Gefühl für das Wesentliche.

Im Mittelpunkt seiner Arbeit stand der Wald in all seinen Erscheinungsformen und seiner vielfältigen Beziehung zur menschlichen Gesellschaft. Mit Leidenschaft hat er sich der Frage des Waldsterbens angenommen und in der bedrohlichen Waldschadensentwicklung eine Krise der Gesellschaft gesehen. Aber er ist nicht nur als Mahner im Kampf gegen das Waldsterben weit über die Grenzen der Schweiz hinaus bekannt geworden; sein forschungspolitisches Engagement und seine menschlichen Fähigkeiten haben ihm weltweit großes Ansehen in der forstlichen Forșchung gebracht. So war es fast zwingend, daßer 1981 in den Vorstand der Internationalen Vereinigung forstlicher Forschungsanstalten (JUFRO) berufen wurde.

5 Jahre lang hat WALTER BOSSHARD auch in der Herausgebergemeinschaft des Forstwissenschaftlichen Centralblattes mitgewirkt und viel zur Gestaltung dieser Zeitschrift beigetragen.

Die Herausgebergemeinschaft des Forstwissenschaftlichen Centralblattes und die Verlagsbuchhandlung Paul Parey gedenken W $A$ i TFR BOSSHARDs mit großem Respekt und tiefer Dankbarkeit.

Die Herausgebergerneinschaft

Ulrich AMMer, Werner Kroth

Karl E. RfHFuess, Walter SCHÖPFER

Peter Schutt, BerNhard Ulrich
Die Verlagsbuchhandlung

Friedricil Grorgl, Rlidol. Grorgi 